\title{
From disks to planets: observational insights
}

\author{
Andrea Isella \\ Physics \& Astronomy Department, Rice University, \\ MS-108, 6100 Main St., Houston, TX, USA, 77005 \\ email: isella@rice.edu
}

\begin{abstract}
The unprecedented sensitivity and imaging capabilities offered by the Atacama Large Millimeter Array (ALMA) are transforming our understanding of protoplanetary disks and, hence, of planet formation. In this brief chapter, I first discuss the main results and caveats related to the measurement of the mass of solids in protoplanetary disks based on millimeterwave observations. I then present a recent analysis of the ALMA observations of the HL Tau disk, which suggests that the observed circular rings might be due to the tidal interaction between Saturn mass planets and the circumstellar material. In the conclusion, I argue that the existing observations of protoplanetary disks suggest that planets might form very early on, perhaps at the same time as the formation of the disk itself.
\end{abstract}

Keywords. Protoplanetary disks, Planets and satellites: formation, Submillimeter: planetary systems, stars: formation

\section{Introduction}

In the last few years, the study of the formation of planets has been revolutionized by the unprecedented imaging capabilities of new infrared and microwave observing facilities. In particular, the Atacama Large Millimeter Array (ALMA) is now capable of delivering high fidelity images at an angular resolution of a few tens of milliarcseconds, which resolve the submillimeter emission from nearby protoplanetary disks on scales as small as $1 \mathrm{AU}$.

ALMA observations of protoplanetary disks are revealing surprising structures which are thought to be caused by the tidal interaction between yet unseen giant planets and the circumstellar material. These observations probe the earliest stages of the formation of new planetary systems and enable us to study the physical processes responsible for the assembly of planets.

At the same time, the superior sensitivity of ALMA observations allows to quickly measure millimeter fluxes for hundreds of protoplanetary disks in the nearest star forming regions. These measurements can be used to estimate the amount of gas and dust in protoplanetary disks and study how the density of the circumstellar material depends on the properties of the host star.

In this brief paper, I first review the main results concerning the measurement of the amount of solids in protoplanetary disks and then discuss a recent analysis of the ALMA observations of HL Tau. A discussion about how these observations inform about the formation of planets is presented in the Conclusions.

\section{Amount of solids in protoplanetary disks: caveats and results}

The study of the structure and evolution of protoplanetary disks mostly relies on photometric measurements of their continuum emission, which, at wavelengths spanning from the near-infrared to the microwave regimes, originates from the thermal emission of solid particles. If the column density of such particles is low, then the continuum emission 
is optically thin, and it can be used to measure the column density of particles themselves. In the opposite regime, i.e., when the column density of solids is high, the continuum emission is optically thick and probes instead the temperature of the $\tau \simeq 1$ layer. For the typical densities of protoplanetary disks, the transition between the optically thick and thin regimes happens between the wavelength of $1 \mathrm{~mm}$ and $1 \mathrm{~cm}$.

The flux density of the optically thin continuum emission at a wavelength $\lambda$ depends on the mass of solids $M_{s}$ through the relation

$$
F(\lambda)=M_{s} \times \kappa_{s}(\lambda) \times B\left(\lambda, T_{s}\right) / d^{2},
$$

where $\kappa_{s}(\lambda)$ is the opacity of the solids, $B\left(\lambda, T_{s}\right)$ is the emission of a black body at the temperature $T_{s}$ of the solids, and $d$ is the distance of the source. Deriving $M_{s}$ from measurements of $I(\lambda)$ therefore requires assumptions on the distance, opacity, and temperature of the solid materials.

The temperature of protoplanetary disks is measured by comparing the disk spectral energy distribution to radiative transfer models. Typical values range from a few hundred degrees Kelvin within 1 AU from solar type stars, to a few degrees Kelvin in the outermost disk regions. The measurement of the disk temperature is affected by relatively small errors (a factor of two at most) and will not be discussed further in this paper. Similarly, the distance of nearby protoplanetary disks relies on parallax measurements that are accurate at a 10\% level, and will not be discussed here.

The opacity of solids is much more uncertain and dominates the overall uncertainty on the measurement of the mass of solids. It can be demonstrated that the solid particles that most efficiently emit thermal radiation at a wavelengths $\lambda$ are those with a radius $a$ such that $2 \pi a=\lambda$. Therefore, the mm- and cm-wave thermal emission mostly arises from particles with radii between about $0.1 \mathrm{~mm}$ and $1 \mathrm{~mm}$, i.e., dust grains. The opacity of these grains can be calculated once the composition and structure is known using, for example, the Mie theory.

However, the population of solids in a protoplanetary disk likely comprises a broader range of sizes. An estimate of the total mass of solids therefore requires assuming a grain size distribution. A common, though not well justified choice, is to a adopt a MRN distribution, $n(a) \propto a^{-3.5}$ (Mathis et al. 1977). In this case, most of the mass is locked into the largest grains $\left(a=a_{\max }\right)$, while most of opacity, and therefore emission, at a wavelength $\lambda$ is provided by the grains with sizes comparable to $\lambda$. It is easy to demonstrate that, in the case of a MRN size distribution, the mass of solids derived from a single wavelength measurement of the flux density at millimeter-wavelengths is proportional to $\sqrt{a_{\max }}$.

Multi-wavelengths observations at wavelengths between about $1 \mathrm{~mm}-1 \mathrm{~cm}$ help in constraining $a_{\max }$. They measure the spectral slope $\alpha$ of the continuum emission $\left[I(\lambda) \propto \lambda^{\alpha}\right]$, which, if the emission is optically thin, is related to the spectral slope of the dust opacity $\beta\left[\kappa(\lambda) \propto \lambda^{-\beta}\right]$, which, in turn, depends on $a_{\max }$. However, the relation between $a_{\max }$ and $\beta$ is uncertain because it depends on the dust composition and size distribution, and, more importantly, it is degenerate for values of $a_{\text {max }}$ larger than a few centimeters (see, e.g., Figure 4 in the PPVI review chapter by Testi et al. 2014). In practice, observations of the thermal dust continuum emission cannot measure the amount of solids locked into particles larger than a few centimeters.

Given all these caveats and assumptions, we should be doubtful about disk mass estimates and, in particular, we should avoid over interpreting mass estimates for single objects. However, mass measurements for homogeneous samples of disks can still provide important clues about the dependence between the mass of solids and the properties of 


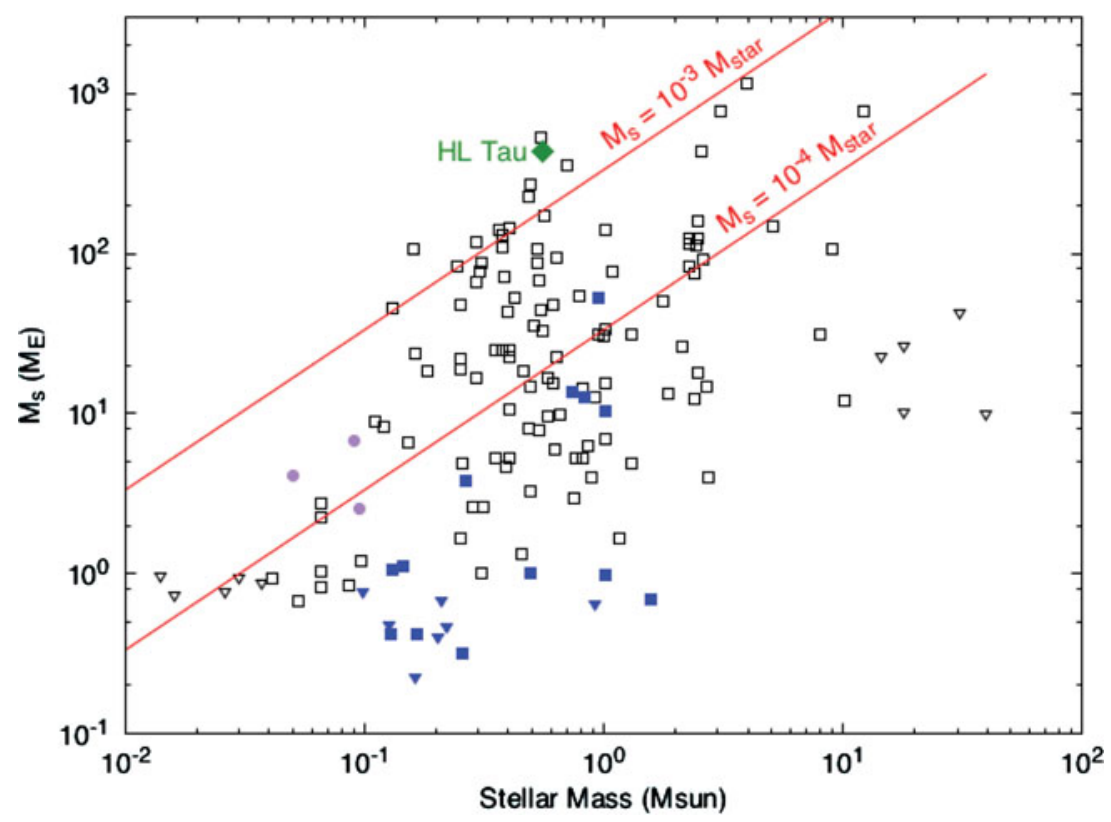

Figure 1. Mass of solids particles in units of the Earth mass measured form millimeter-wave observations of nearly protoplanetary disks as function of the stellar mass. Empty black triangles and squares indicate upper limits and detections, respectively, for disks in Taurus and Ophiuchus (Williams \& Cieza 2011 and references therein). Filled triangles and squares indicate upper limits and detections, respectively, for disks in the Upper Scorpius association (Carpenter et al. 2014). Purple circles indicate disks around brown dwarfs (Ricci et al. 2014). (A color version of this figure is available online.)

the central star (such as age, mass, and metallicity), and help understanding the demographics of planetary systems.

For example, millimeter observations of nearby star forming regions have delivered estimates of the mass of solids for tens of protoplanetary disks spanning ages between 1-10 Myr and masses of the host star between 0.01-30 $\mathrm{M}_{\odot}$ covering stellar spectral types from brawn dwarfs to O-type stars (Figure 1). In a recent review of this topic, Williams \& Cieza (2011) argue that there is a linear correlation between the mass of solids in disks $\left(M_{s}\right)$ and the mass of the central star $\left(M_{\star}\right)$, such that, on average, $M_{s}=10^{-4} \times M_{\star}$. Protoplanetary disks around solar mass stars contain on average about 30 Earth masses $\left(M_{E}\right)$ of solids. As a reference, this value is equal to the minimum mass of solids required to form the planets in our Solar system.

Taken at face value, the relatively low mass of solids measured toward solar type stars suggest that these systems might be able to form only low mass planets. However, one has to keep in mind that this mass estimate is affected by all the uncertainties discussed above, and in particular, by the lack of knowledge about the fraction of mass of solid locked into large $(>10 \mathrm{~cm})$ bodies.

Furthermore, the correlation between the mass of solids and the stellar mass is characterized by a large scatter. For example, the mass of solids around solar mass stars varies by at least two orders of magnitude between 1 and $100 M_{E}$. The lower limit of this range is set by the sensitivity of current observations and it is poised to decrease in the near future as the results of ALMA observations of nearby star forming regions are released (see, e.g., Carpenter et al. 2014, Ricci et al. 2014). Vice versa, the upper limit of the mass range is significant and implies that very few disks contain solids in 
excess of $10^{-3} M_{\star}$, or $300 M_{E}$ for a Solar type star. Although this value is about $10 \times$ the minimum mass required to form the planets in the Solar system, it nevertheless suggests that either the planet formation process is very efficient in converting dust into planets, or that planetesimals, and perhaps planets, are already formed at the age of $1-5 \mathrm{Myr}$.

This result comes with two important caveats. First, Figure 1 shows mass measurements only for Class II disks. Mass estimates for protostellar systems in earlier phases of formation, e.g., Class 0 and I systems, would populate the uppermost part of the diagram which is now mostly empty. Second, as the mass of solids increases, the optical depth of the millimeter-wave emission also increases, and the mass estimates become more uncertain. In particular, if the emission is not completely optically thin, the mass of solids derived from Equation 2.1 provides only a lower limit of the true mass. If protoplanetary disks have the same size and temperature, this optical depth problem becomes more severe as the disk mass increases.

\section{What have we learned from HL Tau?}

A major milestone in the study of protoplanetary disks was achieved in December 2014 with the successful commissioning of the ALMA $10 \mathrm{~km}$ baselines and subsequent release of the dust continuum images of the HL Tau system (ALMA partnership, 2015). After one year from the release of the HL Tau images, the scientific community is debating whether the observed concentric rings are caused by sudden jumps in the radial profile of the dust opacity at radii where the gas temperature drops below the freeze out temperature of the water and CO (Banzatti et al. 2015; Okuzumi et al. 2015; Zhang et al. 2015), or whether the rings are caused by the tidal interaction with unseen planets (Akiyama et al. 2015; Di Pierro et al. 2015; Gonzalez et al. 2015; Picogna et al. 2015). At the time of writing, the planet-disk interaction model is favored because it better reproduces the main observable of the HL Tau system.

In a recent analysis of the ALMA data, Jin et al. (2016) find that the radial profile of the $1 \mathrm{~mm}$ flux density and of the spectral index measured between 0.87 and $1.3 \mathrm{~mm}$ are quantitatively consistent with the tidal interaction between a rather massive disk $\left(M_{d} / M_{\star}=0.13, M_{s} / M_{\star}=0.0013\right)$ and three Saturn mass planets orbiting at about 13 $\mathrm{AU}, 33 \mathrm{AU}$, and $69 \mathrm{AU}$, respectively (Figure 2).

The planets' perturbations on the distribution of gas and dust is shown in the upper panel of Figure 2: the planets open shallow gaps in the gas density and deeper gaps in the distribution of the grains that mostly emits at the wavelength of $1 \mathrm{~mm}$. This difference is due to the fact that, as the planets start opening gaps in the gas density, the submillimeter dust grains located within the gaps radially migrate toward the gap edges. As a result of the planet-disk interaction, the dust-to-gas ratio changes from an initial constant value of 0.01 to about 0.02 in the intra-planets regions, and 0.005 within the gas depleted gaps (top right panel).

Jin et al. (2016) find that most of the $1 \mathrm{~mm}$ continuum emission arising form the HL Tau disk is optically thick (bottom right panel of Figure 2). Theoretical models suggest optical depths between 1 and 3 in the innermost and middle bright rings, respectively, and values larger than a few in the innermost $10 \mathrm{AU}$ from the central star. The estimate of the optical depth results from modeling the dust emission in the regions where the optical depth is less than 1 and needs to be confirmed by observing the HL Tau disk at longer wavelengths. For example, if the analysis of Jin et al. (2006) is correct, the dust emission at $7 \mathrm{~mm}$ from the innermost and middle rings should have optical depth of about 0.4 and 0.2 , respectively. 

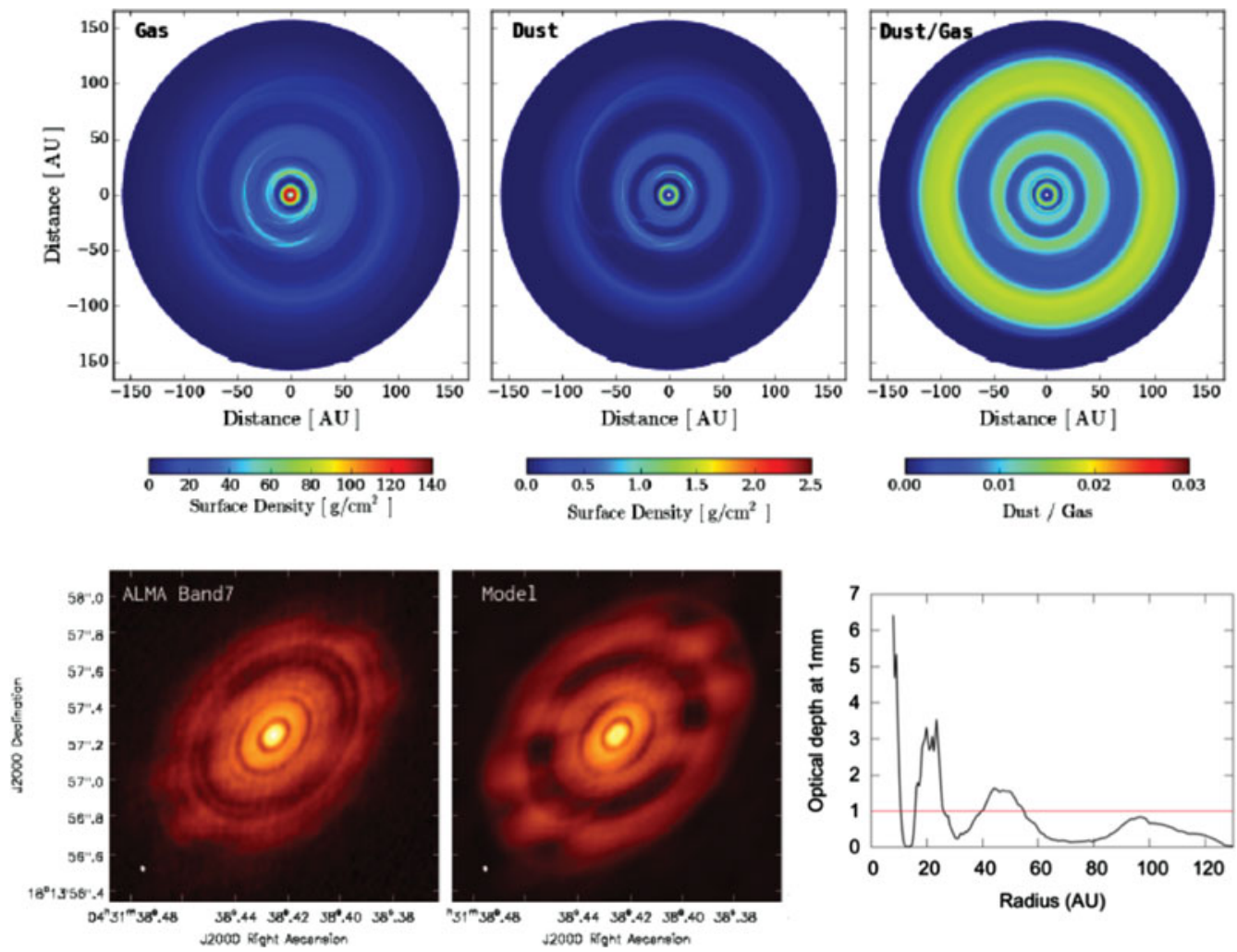

Figure 2. From Jin et al. (2006). Top: model for the gas and dust surface density, as well as dust-to-gas ratio for the HL Tau disk. Concentric rings of dust and gas are caused by the tidal perturbation between the circumstellar material and three Saturn mass planets orbiting at 13 $\mathrm{AU}, 33 \mathrm{AU}$, and $69 \mathrm{AU}$, respectively. Bottom left: comparison between model and observations. The color scale in the two panels is the same and it indicates the flux density. Bottom right: radial profile of the dust opacity at the wavelength of $1 \mathrm{~mm}$ inferred form the observations. ( $A$ color version of this figure is available online.)

Since a large fraction of the $1 \mathrm{~mm}$ continuum emission from the HL Tau disk is optically thick, the estimate of the disk mass is quite uncertain. A similarly large uncertainty likely affects the masses of the other millimeter-bright disks that occupy the top portion of the diagram shown in Figure 1. However, Jin et al. (2016) find that if the HL Tau disk mass were greater than about $0.2 M_{\star}$, the disk would be gravitationally unstable. The measurement of the flux density on one side and dynamical arguments on the other, bracket the total mass of the HL Tau disk between $0.13-0.2 M_{\star}$.

\section{Conclusions}

The relatively small mass of solids measured in the majority of protoplanetary disks with ages between 1-10 Myr, on one side, and the morphology of the HL Tau disk, on the other, suggest that planet formation might start very early on and proceed very quickly, such that the embryos of giant planets already exists in disk younger than 1 Myr. Planet formation might therefore be contemporaneous with the formation of disks themselves, or at least start when the disks are very young, massive, and perhaps gravitationally unstable. 
Gravitational instabilities might explain the existence of Saturn mass planets orbiting at tens of AU from HL Tau. Indeed, gravitational instabilities arise naturally in the cold disk midplane far away from the central star. Furthermore, gravitational instabilities are supposed to be able to form planets much faster than the core accretion model. This would also be consistent with the young age of the HL Tau system. Whereas it might be reasonable to think that planets form when the parent disks are young and massive, it is unclear how the same planets might survive the inward radial migration caused by the interaction with the gaseous disk.

The observations discussed above demonstrate the transforming capabilities of ALMA. In a few years from now, many more systems like HL Tau will be mapped in great details and millimeter fluxes for most of the protoplanetary disks in nearby star forming regions will be measured. At that time, we will be able to derive firmer conclusion on the processes responsible for the formation of planets.

Finally, current ALMA observations stress the need to map protoplanetary disks at centimeter wavelengths in order to peer into the regions that are optically thick at ALMA wavelengths and achieve better estimates of the mass of solid particles. Centimeter-wave observations for the few brightest disks can already be obtained with the Jansky Very Large Array (JLVA), but more sensitive telescopes are required to map the majority of nearby protoplanetary disks. One of such future telescopes is the next generation Vary Large Array (ngVLA), which is discussed in a series of white papers available on arXiv (see, e.g., Carilli et al. 2015, Isella et al. 2015).

\section{Acknowledgement}

A.I. acknowledge support from NSF (grant no. 1535809) and NASA (grant no. NNX15AB06G).

\section{References}

ALMA Partnership, Brogan, C. L., Prez, L. M., Hunter, T. R., Dent, W. R. F., Hales, A. S., Hills, R. E., Corder, S., Fomalont, E. B., Vlahakis, C., Asaki, Y., Barkats, D., Hirota, A., Hodge, J. A., Impellizzeri, C. M. V., Kneissl, R., Liuzzo, E., Lucas, R., Marcelino, N., Matsushita, S., Nakanishi, K., Phillips, N., Richards, A. M. S., Toledo, I., Aladro, R., Broguiere, D., Cortes, J. R., Cortes, P. C., Espada, D., Galarza, F., Garcia-Appadoo, D., Guzman-Ramirez, L., Humphreys, E. M., Jung, T., Kameno, S., Laing, R. A., Leon, S., Marconi, G., Mignano, A., Nikolic, B., Nyman, L.-A., Radiszcz, M., Remijan, A., Rodn, J. A., Sawada, T., Takahashi, S., Tilanus, R. P. J., Vila Vilaro, B., Watson, L. C., Wiklind, T., Akiyama, E., Chapillon, E., de Gregorio-Monsalvo, I., Di Francesco, J., Gueth, F., Kawamura, A., Lee, C.-F., Nguyen Luong, Q., Mangum, J., Pietu, V., Sanhueza, P., Saigo, K., Takakuwa, S., Ubach, C., van Kempen, T., Wootten, A., Castro-Carrizo, A., Francke, H., Gallardo, J., Garcia, J., Gonzalez, S., Hill, T., Kaminski, T., Kurono, Y., Liu, H.-Y., Lopez, C., Morales, F., Plarre, K., Schieven, G., Testi, L., Videla, L., Villard, E., Andreani, P., Hibbard, J. E., \& Tatematsu, K. 2015, ApJ, 808, 3

Banzatti, A., Pinilla, P., Ricci, L., Pontoppidan, K. M., Birnstiel, T., \& Ciesla, F. 2015, ApJ, 815,15

Carilli, C. L., McKinnon, M., Ott, J., Beasley, A., Isella, A., Murphy, E., Leroy, A., Casey, C., Moullet, A., Lacy, M., Hodge, J., Bower, G., Demorest, P., Hull, C., Hughes, M., di Francesco, J., Narayanan, D., Kent, B., Clark, B., \& Butler, B. 2015, eprint arXiv:1510.06438

Carpenter, J. M., Ricci, L., \& Isella, A. 2014, ApJ, 787, 42

Dipierro, G., Price, D., Guillaume, L., Hirsh, K., Cerioli, A., \& Lodato, G. 2015, MNRAS, 453, 73

Gonzalez, J.-F., Laibe, G., Maddison, S. T., Pinte, C., \& Mnard, F. 2015, MNRAS, 454, 36 
Isella, A., Hull, L. H., Moullet, A., Galván-Madrid, R., Johnstone, D., Ricci, L., Tobin, J., Testi, L., Beltran, M., Lazio, J., Siemion, A., Liu, Hauyu B., Du, F., berg, K. I., Bergin, T., Caselli, P., Bourke, T., Carilli, C., Perez, L., Butler, B., de Pater, I., Qi, C., Hofstadter, M., Moreno, R., Alexander, D., Williams, J., Goldsmith, P., Wyatt, M., Loinard, L., Di Francesco, J., Wilner, D., Schilke, P., Ginsburg, A., Snchez-Monge, Zhang, Q., \& Beuther, H. 2015, eprint arXiv:1510.06444

Jin, S., Li, S., Isella, A., Li, H., \& Ji, J. 2016, ApJ in press

Mathis, J. S., Rumpl, W. \& Nordsieck, K. H. 1997, ApJ, 217, 425

Okuzumi, S., Momose, M., Sirono, S., Kobayashi, H., \& Tanaka, H. 2015, ApJ submitted, arxiv: 1510.03556

Picogna, G., \& Wilhelm, K. 2015, A\& A, 584, 110

Ricci, L., Testi, L., Natta, A., Scholz, A., de Gregorio-Monsalvo \& I., Isella, A. 2014, ApJ, 791, 20

Testi, L., Birnstiel, T., Ricci, L., Andrews, S., Blum, J., Carpenter, J., Dominik, C., Isella, A., Natta, A., Williams, J. P., \& Wilner, D. J. 2014, Protostars and Planets VI, 339

Williams, J. P. \& Cieza, L. A. 2011, ARA\&A, 49, 67

Zhang, Ke, Blake, Geoffrey A., Bergin, \& Edwin A. 2015, ApJL, 806, 7 